\title{
Track Paging an Optimal Method for Minimizing Paging and Signaling Cost in Mobile IP
}

\section{Tania Baghaie}

Training Center of Applied Science and Technology, Tehran Municipality Information and Communication Technology Organization, Tehran, Iran.

Email: tb.email@yahoo.com

Received August $8^{\text {th }}, 2013$; revised September $6^{\text {th }}, 2013$; accepted September $13^{\text {th }}, 2013$

Copyright (C) 2013 Tania Baghaie. This is an open access article distributed under the Creative Commons Attribution License, which permits unrestricted use, distribution, and reproduction in any medium, provided the original work is properly cited.

\begin{abstract}
Mobile IP is an Internet standards protocol, which enhances the existing Internet Protocol (IP) to accommodate mobility. In mobile IP protocol, to find the exact location of mobile node for transferring incoming packets to them has a lot of signaling cost. So increasing the performance and decreasing the signaling cost are challenges of this protocol. Using the paging mechanism, its operation is in such a way that it can reduce Relocation update cost and save on power consumption in a mobile node. In addition, it can improve the performance of network by decreasing the need to register an idle mobile node. Mobile IP, currently does not support paging. Therefore, after introducing the mobile IP protocol and reporting related works, this paper will try to introduce a way to add paging support to the Mobile IP. Track paging is proposed to reduce the number of registration message of an idle mobile node to the mobile node's home agent by waiving broad casting method and paging area size. Analytical results show that Track paging can significantly improve the total signaling cost of MIP vs. previous methods. Results also show that specifying the optimal paging area is critical in saving signaling cost of a MIP with paging support.
\end{abstract}

Keywords: Mobile Node; Paging; Registration; Home Agent; Foreign Agent; Binding

\section{Introduction}

Mobile IP (MIP) is a standard proposed by the Internet Engineering Task Force (IETF). MIP was originally designed to serve the needs of globally mobile users who wish to connect their Mobile Node (MN)s to the Internet and maintain connectivity as they move from one place to another, establish new links, and move away from previously established links [1]. MIP enables nodes to change their point of attachment to the Internet without changing their IP address [2]. Multi-step paging has been widely proposed in personal communications services (PCS) systems to reduce the signaling overheads. Similar ideas can be applied to Mobile IP to provide IP paging services. However, current proposed multi-step paging schemes are dependent on paging areas size or the selection of paging sequence or manner of each user [3]. So after introducing the mobile IP protocol and related works, this paper tries to introduce a way that appended paging to the Mobile IP protocol to reduce the number of location updates or registers to the home network, and reduce the signaling cost. The proposed method uses a tracking solution for Paging. In tracking way, when the idle mobile node moved to the new foreign agent, it would let the previous foreign agent know about its new location so this will be continued to the predefined threshold and then mobile node will register its location with the home agent. Many efforts have been made to improve and extend Mobile IP. An effort to enhance the performance of MIP is Route Optimization Protocol [4], which is introduced to solve the problem of triangular routing. Route Optimization is already integrated into MIPv6. Generally, localization is a popular approach to improve the performance of MIP. In wireless mobile networks, paging is a process to determine the exact location of a specific Mobile Terminal (MT) in PCSs or a Mobile Host $(\mathrm{MH})$ in Mobile IP that is in standby state. Paging service is popularly deployed in wireless WAN for two major benefits [5]: to reduce location update cost and to save power consumption of mobile terminals (MT). Paging procedures also generate additional signaling overhead that is paging cost itself and the latency in locating an MH for packet delivery. There exists a tradeoff between 
paging cost and registration cost with respect to PA size. With a bigger paging area (PA) size, an $\mathrm{MH}$ tends to update its location less frequently, thus the location update cost is reduced, but the cost of paging over the PA certainly increases and vice versa. Therefore, it is desirable to figure out the optimal PA size that minimizes the total signaling cost [6].

\section{Related Work}

Mobile IP supports only location registration; however,IP layer paging is needed to reduce signaling overhead while the idle $\mathrm{MH}$ moves from one subnet to another and minimize the power consumption of the MHs. Various authors [5,7-10] have proposed protocols to extend Mobile IP to support paging. Paging is the procedure that allows the network to search for and locate the idle $\mathrm{MH}$ when there is a packet destined for the $\mathrm{MH}$, such that the $\mathrm{MH}$ does not need to register its precise location to the HA when moving within the paging area. An idle MH sends a location registration message to its HA only when it enters a new paging area, rather than when switching from one cell to another. A paging foreign agent (PFA) is a foreign agent that originates the paging request and is responsible for the paging process in its paging area. When the HA needs to contact an idle MH for packet delivery, the HA sends a request to the MH's currently serving PFA, and the PFA in turn pages the $\mathrm{MH}$ in its current paging area [7]. Ramjee et al [5] propose three different paging architectures where the paging agent function is implemented, respectively, on the home agent, domain root router, and the last visited foreign agent. The authors compare the performance of these schemes in terms of the paging load that can be supported and analyze the impact of varying the paging area size. However, a static paging area size is assumed for all MHs. Zhang et al. [10] propose the Paging Extensions for Mobile IP protocol (P-MIP). The analytic mobility model is based on fluid flow. Zhang et al. assume parameters such as the MH's speed, incoming packet rate to be static and the same for all MHs. As a result the paging area size is aggregately configured for all MHs. Castellucia [8] proposes an adaptive and individual paging scheme in which each MH computes its optimal paging area to extend Mobile IP to support paging. Incoming packet rate and mobility parameters are used in analyzing signaling cost. Castellucia's scheme, though, requires frequent computation of the optimal paging area, which impacts power consumption of the MH. Further, a somewhat unrealistic assumption of the scheme, because it uses Euclidean distances in its mobility model, is that an $\mathrm{MH}$ has knowledge of its exact geographical location. Castellucia also calls for protocol modifications that are not compatible with the existing Mobile IP. DIP-MIP [7], a distributed individual paging extension for Mobile IP in IP-based cellular networks is proposed by its own "view" of the network as divided into paging areas - this view being optimized, according to the MH's packet arrival rate and individual mobility pattern, to minimize signaling cost. In DIP-MIP the paging traffic load is distributed to the FAs acting as PFAs in the network. Distribution of the PFA task increases robustness as well by reducing the system's sensitivity to the failure of individual PFAs. We show that DIPMIP can provide significant savings in signaling bandwidth and help IP-based cellular networks to support a large number of MHs. Hierarchical Mobile IPv6 (HMIPv6) [9] complements MIPv6 by facilitating local mobility management. HMIPv6 aims at reducing global signalization and providing improved local mobility management by introducing a hierarchical architecture. Hierarchical mobility management tends to reduce signalization overhead among the mobile node, its correspondent nodes, and its home agent. Indeed, by decomposing the network in several domains managed by a Mobility Anchor Point (MAP), a mobile node does not need to update its correspondent nodes when it moves or roams within the same domain.

\section{Track Paging}

The paging area size is critical in networks that support paging: a high paging cost is generated when the paging area is large, while too small a paging area leads to excessive location registration cost. Optimal performance requires the system to balance these two cost components. Accordingly, track paging proposed to solve this problem by waiving paging area size. According to [11] this method uses the handover and binding ability and tracking the idle mobile node way to send paging message, so it doesn't use broadcasting message. Figure 1 illustrates track paging operation in mobile IP protocol for idle mobile node.

The messages sequence [12] in track paging is another important point that is shown in Figure 2.

Figure 2 illustrate that when the idle mobile node moved between networks and change it access routers (FA) should inform the previous FA to save its current status. This will be help all FAs know to which network the mobile moved.

The base of track paging is FA paging because a paging foreign agent (PFA) is a foreign agent that originates the paging request and is responsible for the paging. Every time the last registered FA were defined as a PFA. The TP model of operation is illustrated in Figures $\mathbf{1}$ and 2 When an MN moves from one network to another one, it performs a home registration with its HA if moved more than threshold this threshold calculated with network administrator [13]. When an MN changes foreignagent (FA) or moved less than threshold, it does not need 
5. Upon receiving packets for $\mathrm{MH}$ that is away, $\mathrm{HA}$

can deduce that the source

has no binding cache for $\mathrm{MH}$

10. Update binding cache

for $\mathrm{MH}$

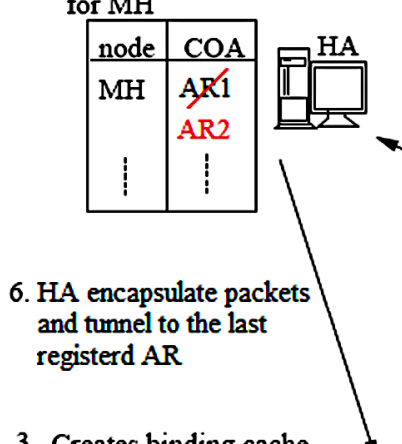

3. Creates binding cache for forwarding

7. Create a Paging request message and check the binding cache for destination

\begin{tabular}{|c|c|}
\hline node & COA \\
\hline $\mathrm{MH}$ & $\mathrm{AR} 2$ \\
& \\
\hline
\end{tabular}

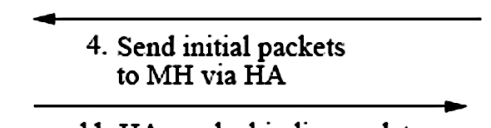

11. HA send a binding update message to $\mathrm{CH}$
12. $\mathrm{CH}$ create binding cache entry for $\mathrm{MH}$
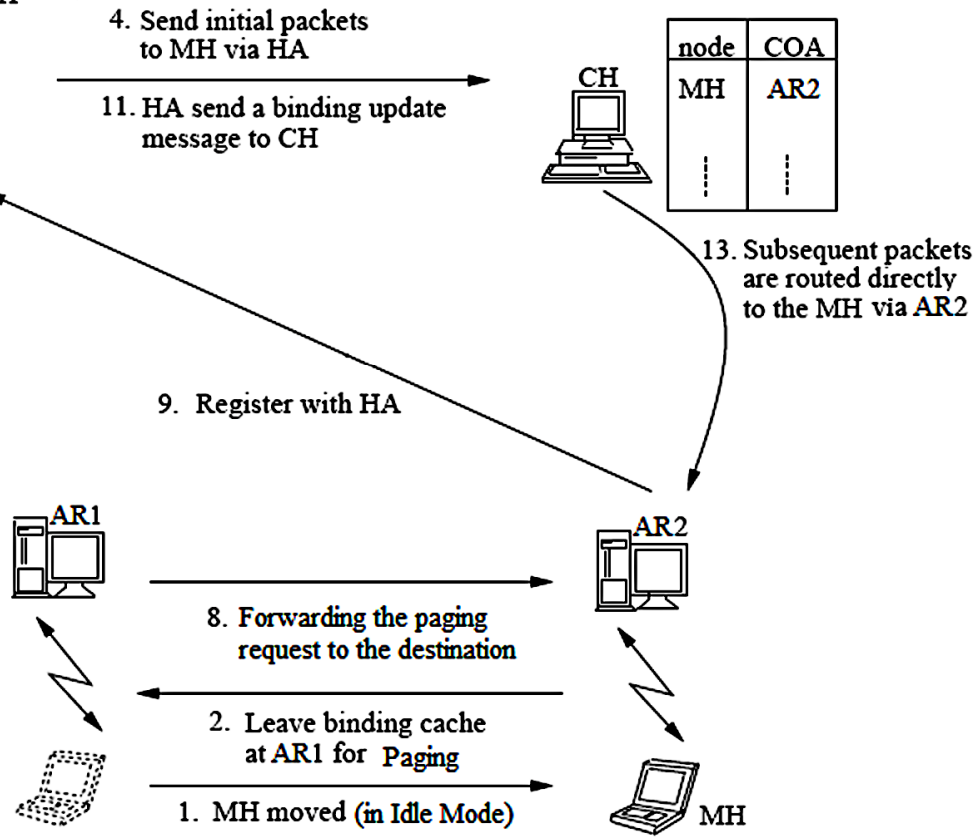

Figure 1. Track paging operation..

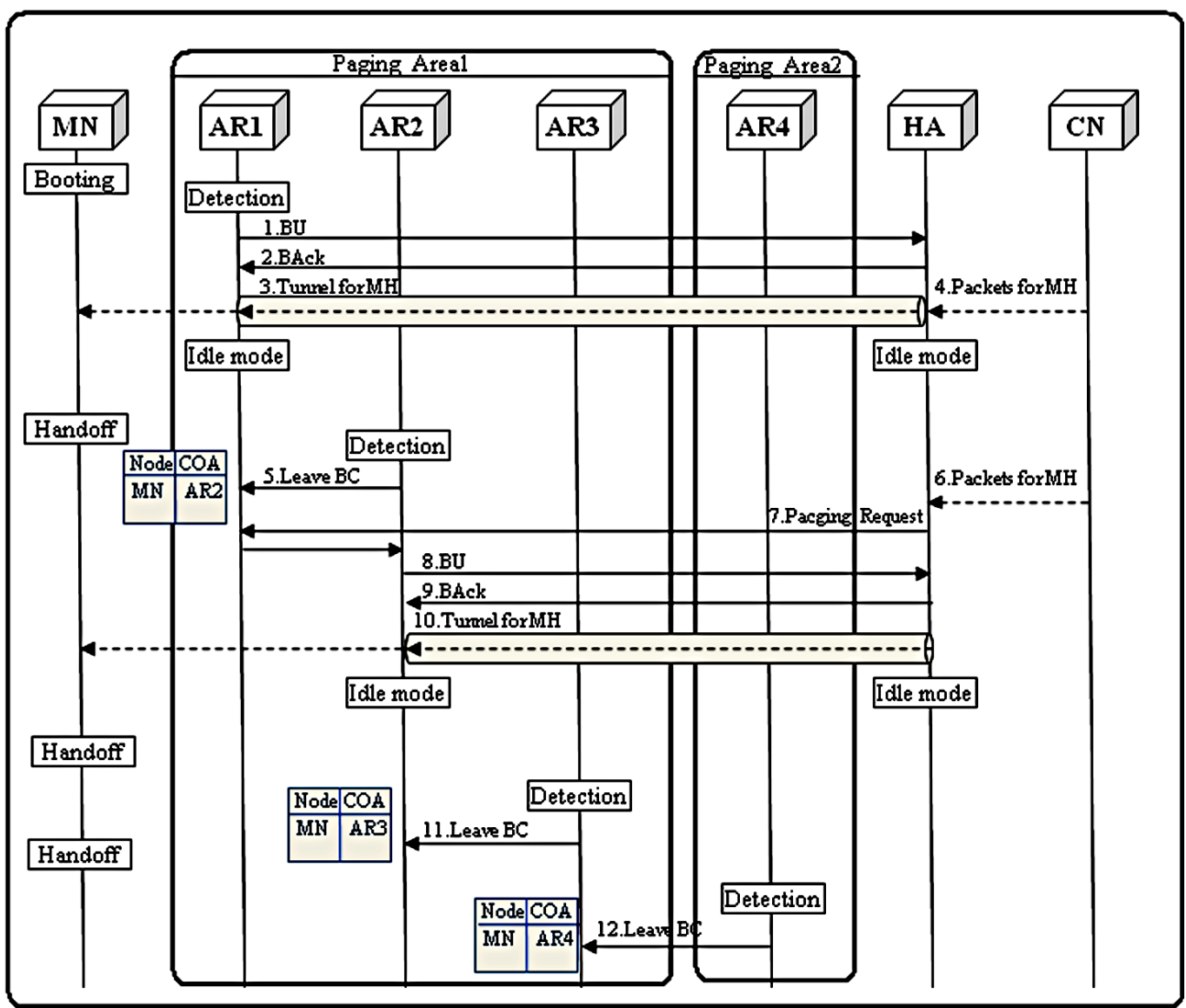

Figure 2. Track Paging. 
to register with its HA. Instead, it performs a binding message to the previous FA to update its FA care-of address. During the communications, packets sent to an $\mathrm{MN}$ are addressed to the HA. The HA encapsulates these packets and sends them to the last registered FA of the MN. The Last foreign agent (LFA) checks its visitor list if find that the mobile node is in idle mode then forward a paging packet to the new FA that is saved on its binding cache. This operation will continued to find the ext. location of Idle mobile node and then mobile node registered with its HA and reply the paging. HA and LFA further relays the buffered packets to the MN. Figure 3 compare the FA paging [6] with TP Paging. Figure shows that to find the direction of mobile node as a usual for each movement to the new area without registration its need to broadcast between all nodes in that area but in second way its need to just follow the way. This will help to decrease the signaling cost especially when $\mathrm{MN}$ moves between the paging areas or move a lot in one paging area.

\section{Signaling Cost Function}

Most of the previous purposed proof the advantages of the domain paging in compare of HA and FA paging. So to valuation the track paging use the similar function to calculate the network signaling cost. Figures 4 and 5 illustrate the location update cost of TP when the movement is equal to threshold an when it is less than threshold.
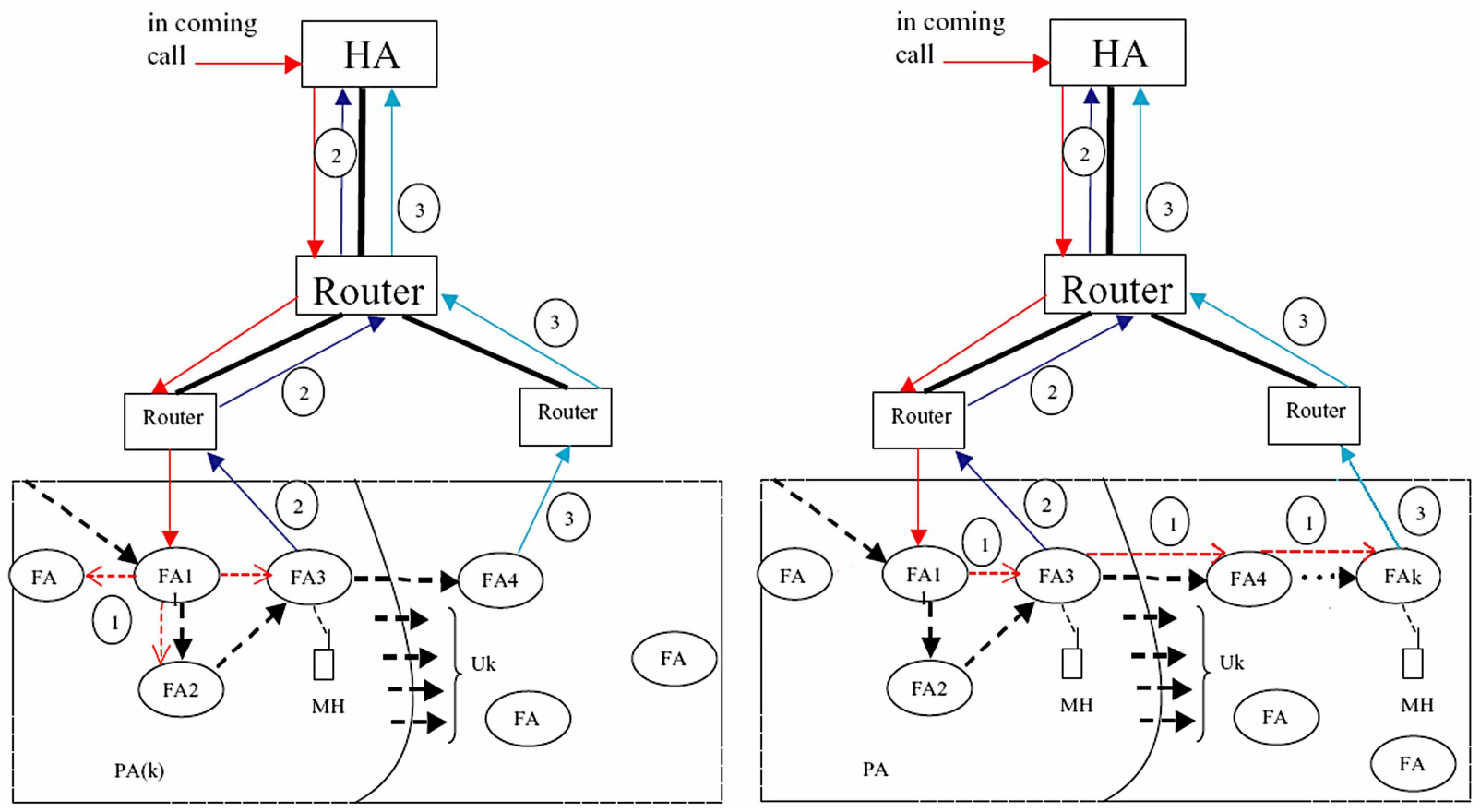

Figure 3. FA paging vs. Track Paging.
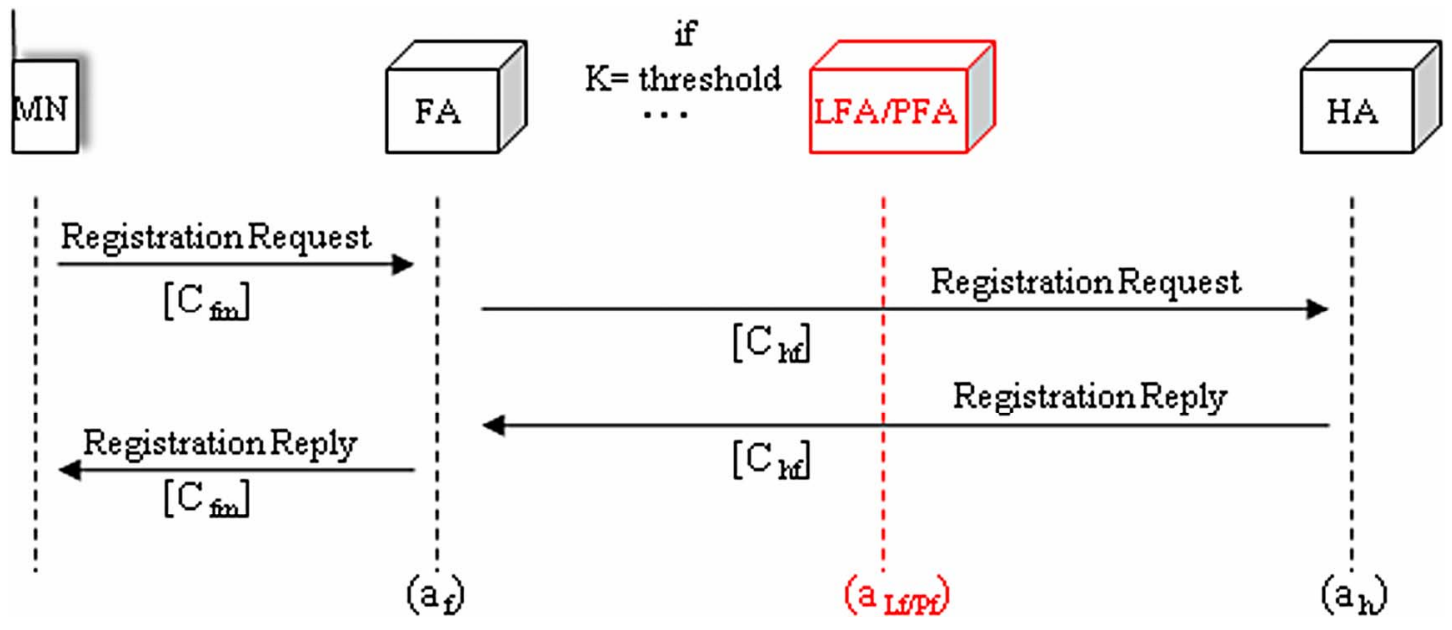

Figure 4. Location update in TP when MN moved equal to threshold. 

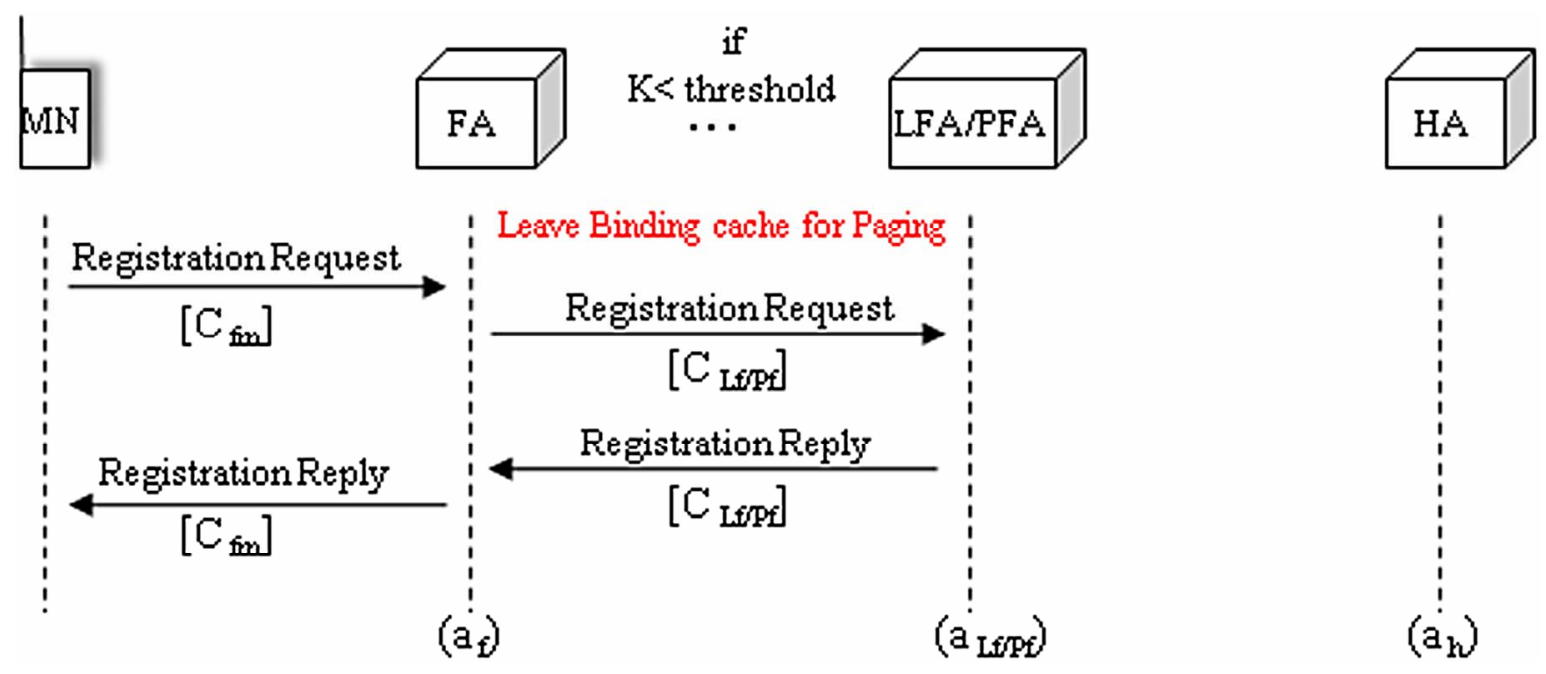

Figure 5. Location update in TP when MN moved less than threshold.

\subsection{Location Update Cost}

Similar to [11], we define the following parameters for location update:

$C_{h f}$ : The transmission cost of location update between the HA and the FA.

$C_{l f / p f}$ : The transmission cost of location update between the LFA and the previous FA.

$C_{f m}$ : The transmission cost of location update over the wireless link between the FA and the MN.

$a_{h}$ : The processing cost of location update at the HA.

$a_{f}$ : The processing cost of location update at the GFA.

$a_{l f / p f}$ : The processing cost of location update at the LFA or PFA.

If assume each MN may move randomly between $N$ subnets and there are $K$ is a threshold of this network. We propose a discrete system to model the movements of each MN. In our model, MNs may visit a subnet more than once and it may also move back and forth between two subnets. We call the action each MN moving out of a subnet a movement.

According to these message flows, the home registration cost for each location update when threshold $=K$ can be calculated as (e.g., see Equation (1)).

$$
C_{U h}=2 a_{f}+a_{h}+2 C_{h f}+2 C_{f m}
$$

In the other hand the previous FA registration cost for each location update when threshold $>K$ can be calculated as (e.g., see Equation (2)).

$$
C_{U H}=2 a_{f}+a_{L f / P f} 2 C_{f m}+2 C_{L f / P f}+2 C_{f m}
$$

If Let $l_{h-l f}$ be the average distance between the HA and the LFA in terms of the number of hops packets travel, and also $l_{l f-p f}$ be the average distance between the LFA and the Previous FA. We assume the transmission cost is proportional to the distance between the source and the destination mobility agents and the proportionality con- stant is $\delta_{U}$. Since the transmission cost of the wireless link is generally higher than that of the wired link so assume that the transmission cost over the wireless link is $\rho$ times higher than the unit distance wire line transmission cost. The transmission cost between the FA and the MN can be written as (e.g., see Equation (3)).

$$
C_{f m}=\rho \delta_{U}
$$

Then the home registration and previous FA registration costs for each location update can be expressed as (e.g., see Equation (4)):

$$
\begin{gathered}
C_{U h}=2 a_{f}+a_{L f / P f}+2\left(l_{h f}+\rho\right) \delta_{U} \\
C_{U H}=2 a_{f}+a_{L f / P f}+2\left(l_{L f / P f}+\rho\right) \delta_{U}
\end{gathered}
$$

Here define a random variable $M$ so that each MN moves out of a threshold limitation at $M$ movement. At movement 1 , MNs may reside in either subnet $1,2, \cdots$ or $\mathrm{N}$. At movement 2, MNs may move to any of the $\mathrm{N}$ subnets. We assume MNs will move out to the $N-t$ (threshold) other subnets with equal probability $\frac{1}{N-t}$.

The probability of performing a home registration atmovement $m$ is (e.g., see Equation (5)):

$$
\begin{aligned}
& P_{h}^{m}=\frac{N}{N-1} \cdot\left(\frac{1}{N-1}\right)^{m-2}=N\left(\frac{1}{N-t}\right)^{m-1}, \\
& \text { where } 2 \leq m<t=\text { thershold }
\end{aligned}
$$

It can be shown that the expectation of $M$ is (e.g., see Equation (6)):

$$
E[M]=\sum_{m=2}^{\text {thershold }} m P_{h}^{m}=1+\frac{N-t}{N}
$$

Assume the average time each MN stays in each subnet before making a movement is $T_{f}$. Therefore, the location update cost per unit time is (e.g., see Equation (7)): 


$$
C L U=\frac{E[M] C U H+C U h}{E[M] T f}
$$

\subsection{Packet Delivery Cost}

Packet delivery cost includes below parameters:

$T_{h-l f}$ : The transmission cost of packet delivery between the HA and the LFA.

$T_{h-l f}$ : The transmission cost of packet delivery between the Previous FA and the LFA.

$u_{h}$ : The processing cost of packet delivery at the HA.

$u_{l f}$ : The processing cost of packet delivery at the GFA.

The cost of each packet delivery procedure can be expressed as (e.g., see Equation (8)):

$$
C_{P D}=u_{h}+u_{l f}+T_{l f-p f}+T_{h-l f}
$$

We assume the transmission cost of delivering data packets is proportional to the distance between the sending and the receiving mobility agents with the proportionality constant $\delta_{D}$.

Then $T_{l f-f}=l_{l f-p f} \delta_{D}$ and $T_{h-l f}=l_{h-l f} \delta_{D}$

Where $\lambda_{\alpha}$ is the average packet arrival rate for each $\mathrm{MN}, \alpha$ and $\beta$ are weighting factors of visitor list and routing table lookups, $\eta$ is a packet delivery processing cost constant so The processing cost function at the HA can be as: $v_{h}=\eta \lambda_{a}$ Then the total packet delivery cost per unit time is (e.g., see Equation (9)):

$$
C_{P D}=\eta \lambda_{a}+\left(l_{h-l f}+l_{l f-f}\right) \delta_{D}
$$

\subsection{Total Signaling Cost}

Based on the above analysis, we may get the overall average [11]. (e.g., see Equation (10))

$$
C_{T O T}=C_{L U}+C_{P D}
$$

\section{Analytical Model}

In this section, we demonstrate some numerical results. Table 1 lists some of the parameters used in our performance analysis. Since the total number of subnets that MNs may access through wireless channels is limited, we assume $N=30$. For our numerical evaluation, we assume $l_{h-l f}=25$ and $l_{l f-f}=10$ are fixed numbers.

At first we Impact of Residence Time on the Total

\begin{tabular}{|c|c|c|c|c|c|c|c|c|c|}
\hline \multicolumn{3}{|c|}{$\begin{array}{c}\text { Pkt. } \\
\text { Process } \\
\text { Cost }\end{array}$} & \multicolumn{2}{|c|}{$\begin{array}{l}\text { Distance } \\
\text { Cost Unit }\end{array}$} & \multirow{2}{*}{$\begin{array}{c}\text { Wireless } \\
\text { Multiple } \\
\boldsymbol{\rho}\end{array}$} & \multirow{2}{*}{$\begin{array}{c}\text { \# of } \\
\text { MNs/subnet } \\
\omega\end{array}$} & \multicolumn{2}{|c|}{ Weight } & \multirow{2}{*}{$\begin{array}{c}\text { Pkt } \\
\begin{array}{c}\text { Delivery } \\
\text { Const. }\end{array} \\
\boldsymbol{\eta}\end{array}$} \\
\hline ah & ah & & $\delta_{U}$ & $\delta_{D}$ & & & $\alpha$ & $\boldsymbol{\beta}$ & \\
\hline 30 & 20 & 15 & 0.2 & 0.5 & 10 & 15 & 0.3 & 0.7 & 10 \\
\hline
\end{tabular}
Signaling Cost, and compare it with Regional Registration method a kind of using domain paging mechanism.

Table 1. Margin specifications.
Figures 6 and 7 show that the TP method is better than the RR method.

And then we The Impact of Packet Arrival on the Total Signaling Cost, and compare it with Regional Registration method a kind of using domain paging mechanism. Figures 8 and 9 show that the TP method is better than the RR method.

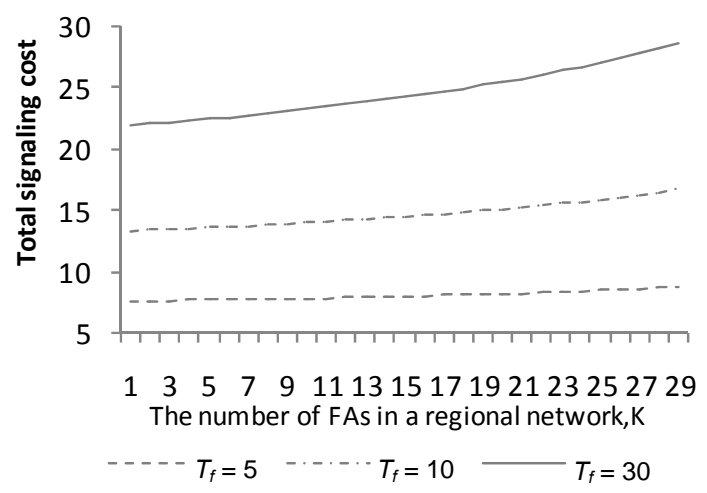

Figure 6. Total signaling cost with various $T_{f}$ in Track paging.

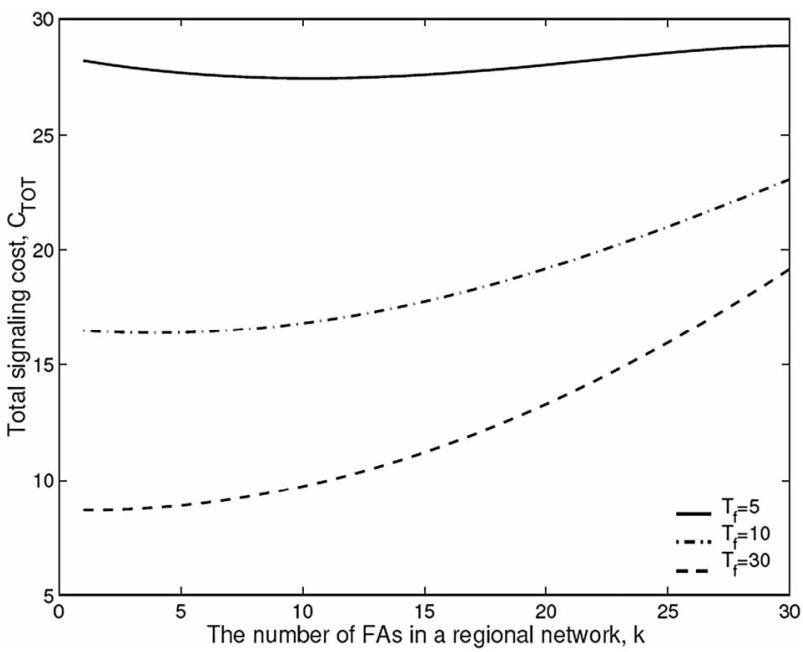

Figure 7. Total signaling cost with various $T_{f}$ in Regional Registration [11].

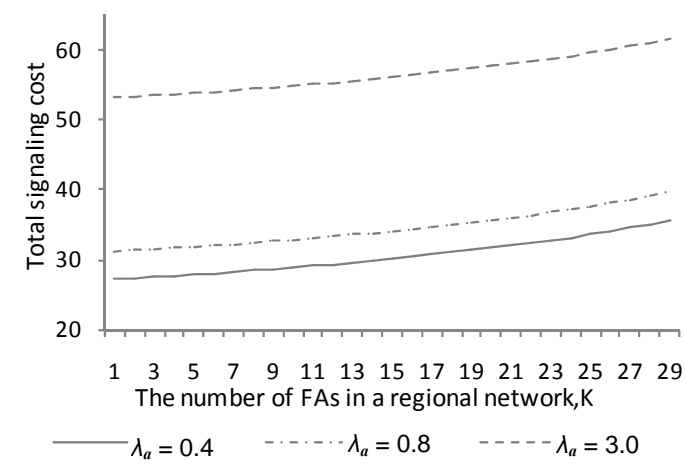

Figure 8. Total signaling costwith various $\lambda_{a}$ in Track paging. 


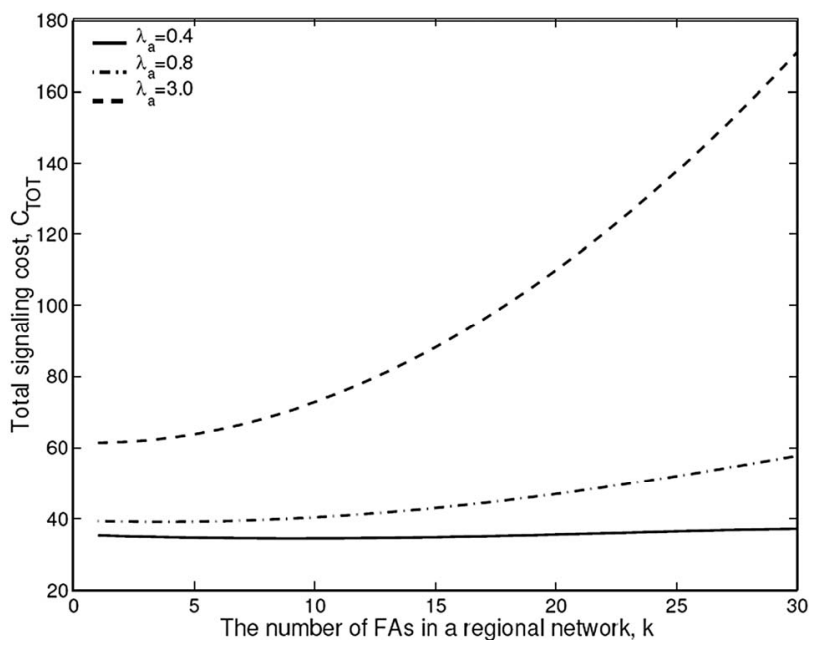

Figure 9. Total signaling cost with various $\lambda_{a}$ in Regional Registration [11].

\section{Conclusion}

In this paper, we introduced Track Paging, an optimal Method for Minimizing Paging and Signaling Cost for Mobile IP which results in the minimum consumption of network resource. The mobility of MNs is modeled by a analytical model. Based on this model, we obtained the average signaling cost function of location update and packet delivery. Given this average total cost function, we proof that the paging area size is less efficient than other methods. Our analytical model does not have any constraint on the shape and the geographic location of Internet subnets. In the future works we will investigate how to find the direction of mobile node with local Registration and signaling instead of registration between mobile host and mobile node.

\section{REFERENCES}

[1] D. Saha, A. Mukherjee, I. S. Misra and M. Chakraborty, "Mobility Support in IP: A Survey of Related Protocols," IEEE Network, Vol. 18, No. 6, 2004, pp. 34-40. http://dx.doi.org/10.1109/MNET.2004.1355033

[2] C. Perkins, "IP Mobility Support for IPv4," 2002. http://www.rfc-editor.org/rfc/rfc3344.txt
[3] J. Xie, "User Independent Paging Scheme for Mobile IP," Wireless Networks, Vol. 12, No. 2, 2006, pp. 145-158. http://dx.doi.org/10.1007/s11276-005-5262-2

[4] J. Mangues, et al., "IP Mobility: Macromobility, Micromobility, Quality of Service and Security," Journal of the Council of European Professional Informatics Societies, Vol. 5, No. 1, 2004, pp. 49-55.

[5] R. Ramjee, L. Li, T. La Porta and S. Kasera, "IP Paging Service for Mobile Hosts," Wireless Networks, Vol. 8, No. 5, 2002, pp. 332-344.

[6] H. Tuan Do and Y. Onozato, "A Comparison of Different Paging Mechanisms for Mobile IP," Wireless Networks, Vol. 13, No. 3, 2007, pp. 379-395. http://dx.doi.org/10.1007/s11276-006-6467-8

[7] C. Chuon and S. Guha, "DIP-MIP: Distributed Individual Paging Extension for Mobile IP in IP-Based Cellular Networks," Computer Communications, Vol. 30, No. 6, 2007, pp. 1178-1186. http://dx.doi.org/10.1016/j.comcom.2006.12.002

[8] C. Castelluccia, "Extending Moile IP with Adaptive Individual Paging: A Performance Analysis," ACM SIGMOBILE Mobile Computing and Communications Review, Vol. 5, No. 2, pp. 14-26.

[9] S. Pack and Y. Choi, "A Study on Performance of Hierarchical Mobile IPv6 in IP-Based Cellular Network," IEICE Transactions on Communications, Vol. E87B, No. 3, 2004, pp. 462-469.

[10] X. Zhang, J. G. Castellanos and A. T. Campbell, "P-MIP: Paging Extensions for Mobile IP," Mobile Networks and Applications, Vol. 7, No. 2002, pp. 127-141.

[11] J. Xie and I. F. Akyildiz, "An Optimal Location Management Scheme for Minimizing Signaling Cost in Mobile IP," ICC 2002 IEEE International Conference on Communications, Vol. 5, 2002, pp. 3313-3317.

[12] J. H. Lee, T. M. Chung, S. Pack and S. Gundavelli, "Shall We Apply Paging Technologies to Proxy Mobile IPv6?" Proceedings of the $3 r d$ International Workshop on Mobility in the Evolving Internet Architecture, Washington, August 2008, pp. 37-42.

[13] J. W. Lee, H. J. Lee and D.-H. Cho, "Effect of Dormant Registration on Performance of Mobility Management Based on IP Paging in Wireless Data Networks," International Journal of Electronics and Communications, Vol. 59, No. 5, 2005, pp. 319-323. http://dx.doi.org/10.1016/j.aeue.2004.11.009 